\title{
Association Between Regorafenib Dose and Efficacy Against Metastatic Colorectal Cancer in a Real- world Setting: A Retrospective Study
}

\section{Masahiro Hatori ( $\sim$ masahiro.hatori@jfcr.or.jp )}

Department of Pharmacy, Cancer Institute Hospital, Japanese Foundation for Cancer Research, 3-10-6 Ariake, Koto-ku, Tokyo 135-8550, Japan

Kazuyoshi Kawakami

Department of Pharmacy, Cancer Institute Hospital, Japanese Foundation for Cancer Research

\section{Takeru Wakatsuki}

Department of Gastroenterological Chemotherapy, Cancer Institute Hospital, Japanese Foundation for Cancer Research

\section{Eiji Shinozaki}

Department of Gastroenterological Chemotherapy, Cancer Institute Hospital, Japanese Foundation for Cancer Research

\section{Kazuo Kobayashi}

Department of Pharmacy, Cancer Institute Hospital, Japanese Foundation for Cancer Research

\section{Takeshi Aoyama}

Department of Pharmacy, Cancer Institute Hospital, Japanese Foundation for Cancer Research

\section{Yasuhiro Nakano}

Department of Pharmacy, Cancer Institute Hospital, Japanese Foundation for Cancer Research

\section{Kenichi Suzuki}

Section for Practical Education, Hoshi University School of Pharmacy and Pharmaceutical Sciences

\section{Kensei Yamaguchi}

Department of Gastroenterological Chemotherapy, Cancer Institute Hospital, Japanese Foundation for Cancer Research

\section{Toshihiro Hama}

Department of Pharmacy, Cancer Institute Hospital, Japanese Foundation for Cancer Research

\section{Research Article}

Keywords: Regorafenib, Cumulative dose, Real-world, Prognostic factor

Posted Date: December 10th, 2020

DOI: https://doi.org/10.21203/rs.3.rs-122412/v1 
License: (c) (i) This work is licensed under a Creative Commons Attribution 4.0 International License. Read Full License 


\section{Abstract}

Background: The association between regorafenib dosage in the treatment of metastatic colorectal cancer and efficacy is currently not well established. It was previously reported that the regorafenib dose as prescribed is associated with efficacy, but doses in actual clinical settings have not been analyzed. This study was to examine the association between the clinical significance of the cumulative dose of regorafenib in the early cycles and treatment efficacy in patients with metastatic colorectal cancer.

Methods: We retrospectively analyzed patients with metastatic colorectal cancer who had received regorafenib as third-line or later chemotherapy between May 2013 and June 2018. Patients who were not treated in the Pharmaceutical Outpatient Clinic for compliance assessment were excluded. Overall survival was calculated using the Kaplan-Meier method. Prognostic factors, including baseline demographics and adverse events, were evaluated using Cox proportional hazard models.

Results: A total of 176 patients were enrolled. By multivariate analysis, total dose until the second cycle $<3180 \mathrm{mg}$ (HR 1.71, 95\% Cl, 1.20-2.44, $P=0.003$ ), age $<65$ years (HR 1.96, 95\% Cl, 1.36-2.86, $P<0.001$ ), PS 2 (HR 1.81, 95\% Cl, 1.28-2.57, $P=0.001$ ), hepatic metastasis (HR 2.86, 95\% Cl, 1.90-4.30, $P<0.001$ ), and regorafenib initial dose $\leq 120 \mathrm{mg}(\mathrm{HR} 1.71,95 \% \mathrm{Cl}, 1.14-2.58, P=0.01)$ were independent negative predictors of overall survival. Median survival times of the lower dose group ( $<3180 \mathrm{mg})$ and higher dose group ( $\geq 3180 \mathrm{mg})$ were 5.8 and 7.6 months, respectively $(P=0.045)$.

Conclusion: The cumulative dose of regorafenib until the second cycle in patients with metastatic colorectal cancer was associated with survival. It is important to individualize regorafenib dose in metastatic colorectal cancer patients.

\section{Background}

Colorectal cancer is the third most commonly diagnosed cancer, with approximately 1.8 million cases worldwide in 2018 [1]. The outcome of treatment has improved year by year, and the overall survival (OS) of colorectal cancer is longer than that of other cancers. However, metastatic colorectal cancer (mCRC) continues to have high mortality.

Regorafenib is an oral multitargeted kinase inhibitor that targets vascular endothelial growth factor receptor 1-3, tyrosine kinase with immunoglobulin and epidermal growth homology domain 2, KIT, rearranged during transcription tyrosine kinase, rat fibroblastoma 1, v-raf murine sarcoma viral oncogene homolog B1, platelet-derived growth factor receptor, and fibroblast growth factor receptor [2] In the CORRECT trial, regorafenib significantly improved survival in patients with $\mathrm{mCRC}$ who were refractory to standard chemotherapy [3]. Although regorafenib contributes to the survival benefit, the emergence of severe toxicities, such as hand-foot skin reaction (HFSR) and skin rash, have come to limit the continuity of regorafenib. In particular, higher frequencies of adverse events such as severe HFSR and hypertension in the Japanese subpopulation are well known compared to the non-Japanese subpopulation (HFSR: $80 \%$ vs $39.3 \%$, and hypertension $24.6 \%$ vs $1.8 \%$, respectively) [4]. In the REBACCA study, the survival and 
safety profiles of regorafenib in the real-life setting were similar to those reported in prospective trials [5]. Therefore, establishment of an optimal administration method considering efficacy and tolerability is desired.

It is reported that toxicities have caused discontinuation or dose reductions in the use of regorafenib [6, 7]. The cumulative incidence of HFSR and liver dysfunction in a prospective observational study was higher in patients who initially received $160 \mathrm{mg}$ than in those who received $\leq 120 \mathrm{mg}$ [6]. As a result, doseescalation strategies have been attempted. In the ReDOS study, in which the starting dose was $80 \mathrm{mg}$ with weekly dose-escalations up to $160 \mathrm{mg}$ in the dose-escalation group, a higher proportion of patients in the dose-escalation group achieved cycle 3 of treatment compared with the standard-dose group, with numerically longer OS in the dose-escalation group [8]. In the RESET study, with used another doseescalation strategy of a starting dose of $120 \mathrm{mg}$, patients who required dose modification exhibited a better disease control rate. In addition, that study suggested that it is important in achieving disease control to continue treatment within the first 28 days [9]. These data suggest that adjustment of the regorafenib dose is important and that the cumulative dose in the early cycles may be associated with disease control. However, there are no data indicating what total dose of regorafenib would be sufficient to achieve disease control. In this study, we measured the cumulative dose of regorafenib (i.e., the actual dose taken by patients within the initial 2 cycles) and examined the relationship between the cumulative dose of regorafenib and survival in a real-world setting. The aim of this study was to examine the association between the clinical significance of the cumulative dose of regorafenib in the early cycles and treatment efficacy in patients with $\mathrm{mCRC}$.

\section{Methods}

\section{Study population}

All patients who were treated with regorafenib at the Cancer Institute Hospital between May 2013 and June 2018 were enrolled. Exclusion criteria for this retrospective study included: (1) diagnosis of gastrointestinal stromal tumor; (2) enrollment in another clinical trial; (3) unclear duration of regorafenib administration because the patient transferred to another hospital; (4) patients who were not treated in the Pharmaceutical Outpatient Clinic (POC) for compliance assessment. The clinical protocol was approved by the Institutional Review Board of the Cancer Institute Hospital (approval number 2018-1239).

\section{Treatment}

Regorafenib was administered orally as third-line or later chemotherapy. The standard dose was 160 $\mathrm{mg}$ /day daily for the first 21 days of a 28-day cycle. Treatment continued until disease progression, intolerable toxicity, or patient refusal. In this study, cumulative dose until the second cycle was defined as the amount of regorafenib that patients took until day 56 , because some patients experienced an irregular schedule due to delays or interruptions.

\section{Data collection}


We gathered the following demographic data: age, gender, Eastern Cooperative Oncology Group (ECOG) performance status (PS), primary colorectal site, metastatic site (peritoneum, liver, lung), number of metastatic sites, site of primary tumor, history of adjuvant chemotherapy, number of prior chemotherapy sessions, use of antibody drugs, regorafenib initial dose, KRAS mutations, and history of trifluridine/tipiracil (FTD/TPI) use. We collected data regarding adverse events related to regorafenib: HFSR, liver dysfunction, hypertension, skin rash, and emergency hospitalization. The severity of adverse events was evaluated according to the National Cancer Institute Common Terminology Criteria for Adverse Events (NCl-CTCAE) 4.0 [10]. We retrospectively collected these data from electronic medical records. In addition, we calculated the cumulative dose of regorafenib and evaluated adherence to regorafenib using pill counts and patient-reported treatment diaries of the POC, as previously reported [11].

\section{Statistical analysis}

OS was defined as the time from initiation of regorafenib administration to death from any cause. OS was calculated using the Kaplan-Meier method, and differences were evaluated using the log-rank test. The study population was separated into two groups by median regorafenib total dose until the second cycle (one group consisting of patients with total dose $\geq 3180 \mathrm{mg}$ and the other with median dose $<3180$ $\mathrm{mg}$ ) in order to evaluate OS and adverse events.

Pearson's chi-square test or Fisher's exact test was used to compare patient characteristics and adverse events. Univariate and multivariate analyses were performed to evaluate prognostic factors using Cox proportional hazard models. We selected factors with substantial impacts $(P<0.2)$ in the univariate analysis and previously reported prognostic factors. These were subsequently evaluated by multivariate analysis. We considered differences to be significant when the $P$ value was $<0.05$, and all tests were twosided. SPSS software, version 24 (IBM Corp., Armonk, NY, USA), was used for all statistical analyses.

\section{Results}

\section{Patient characteristics}

A total of 197 patients were enrolled, and 21 patients were excluded because they transferred to another hospital $(n=20)$ or enrolled in another clinical trial $(n=1)$. Consequently, 176 patients were evaluated in this study. Patient characteristics are listed in Table 1. The vast majority of patients were PS 0 or 1 (91.7\%); almost $70 \%$ of patients had a left-sided tumor, and almost half of the patients were KRAS wild type. More than $80 \%$ of patients received regorafenib as third- or fourth-line chemotherapy, and the vast majority of patients received fluoropyrimidine, irinotecan, oxaliplatin, and bevacizumab. Almost $70 \%$ of patients received regorafenib at an initial dose of $160 \mathrm{mg}$, and the remaining patients $(29.7 \%)$ received a lower dose.

\section{OS and analysis of prognostic factors}


The median follow-up period from starting regorafenib to enrollment was 4.45 years among the 176 patients included in the study. The median OS time was 6.7 months $(95 \% \mathrm{Cl}, 5.74-7.64$ months). The regorafenib median cumulative dose was $3180 \mathrm{mg}$. In the multivariate analysis, total dose until the second cycle $<3180 \mathrm{mg}$ (HR 1.71, 95\% Cl, 1.20-2.44, $P=0.003$ ), age $<65$ years (HR 1.96, 95\% Cl, 1.36-2.86, $P<0.001$ ), PS 2 (HR 1.81, 95\% Cl, 1.28-2.57, $P=0.001$ ), hepatic metastasis (HR 2.86, 95\% Cl, 1.90-4.30, $P$ $<0.001)$, and regorafenib initial dose $\leq 120 \mathrm{mg}(\mathrm{HR} 1.71,95 \% \mathrm{Cl}, 1.14-2.58, P=0.01)$ were extracted as statistically significant independent poor prognostic factors (Table 2). HFSR was not extracted as a prognostic factor $(P=0.325)$.

OS curves were clearly separated according to the cumulative dose of regorafenib within the initial two cycles (Fig. 1). Median survival times of the lower-dose group ( $<3180 \mathrm{mg}$ ) and higher-dose group ( $\geq 3180$ $\mathrm{mg}$ ) were 5.8 and 7.6 months, respectively $(P=0.045)$. We also compared the patient characteristics between the two groups (Table 3$)$. Gender $(P=0.011)$ and adjuvant chemotherapy $(P=0.023)$ were statistically skewed between groups. However, they were not identified as prognostic factors in the multivariate analysis.

\section{Adverse events related to regorafenib}

We examined whether adverse events caused a reduction in cumulative regorafenib dose. Patients could be separated into two groups based on the frequency of primary adverse events (Table 4). All grades of skin rash were reported in 7 patients $(7.7 \%)$ in the higher-dose group and 17 patients (20\%) in the lowerdose group. Emergency hospitalization was reported for 5 patients $(5.5 \%)$ in the higher-dose group and 16 patients $(18.8 \%)$ in the lower-dose group. All grades of HFSR $(P=0.01), \geq$ grade 3 hypertension $(P=$ $0.008)$, all grades $(P=0.017)$ and $\geq$ grade $3(P=0.018)$ skin rash, and emergency hospitalization $(P=$ $0.006)$ were statistically significant. Liver dysfunction was not statistically significant regardless of grade.

\section{Discussion}

Our multivariate analysis identified total dose until the second cycle $<3180 \mathrm{mg}$, age $<65$ years, PS 2 , hepatic metastasis, and regorafenib initial dose $\leq 120 \mathrm{mg}$ as prognostic factors of regorafenib. In groups divided by median dose, regorafenib total dose was associated with OS. It should be noted that a particular cut-off value for cumulative regorafenib dose was presented because it was not reported previously.

In this study, patients who dropped-out early due to adverse events or progressive disease, and we therefore considered the potential for confounding bias. We examined the study population except for early drop-out cases in which patients discontinued treatment until cycle 2 because of severe adverse events or progressive disease in the same multivariate analysis. In exception cases, total dose until the second cycle <3180 mg (HR 1.97, 95\% Cl, 1.00-3.86, $P=0.0496)$ was extracted as a statistically significant independent poor prognostic factor (Table S2). These results clearly demonstrate the clinical 
significance of the cumulative dose of regorafenib in the early cycles with regard to treatment efficacy in patients with $\mathrm{mCRC}$.

A total of 122 of 176 patients (69.3\%) in this study were treated with regorafenib at an initial dose of 160 $\mathrm{mg}$ because the study duration ranged from the time regorafenib went on the market to the close of observation. However, the number of patients treated with an initial dose $\leq 120 \mathrm{mg}$ is currently increasing as a means of preventing discontinuation due to intolerable toxicity. In a recent meta-analysis, treatment with regorafenib at the standard dose of $160 \mathrm{mg}$ was associated with a significant increase in adverse events related to permanent discontinuation, dose interruptions, and dose reductions [12]. Optimizing treatment by means such as personalizing the regorafenib dose and schedule adjustments is common in clinical practice, and many physicians have adopted an empirical approach to manage toxicity as a result of phase III studies [13]. A recent observational cohort study suggested that individualized dosing strategies in patients with mCRC might lead to improved clinical outcomes [14]. In the CORRELATE prospective observational study, the regorafenib toxicity profile was similar to that reported in phase III trials. The starting dose for almost half of the patients in that study was less than the approved $160 \mathrm{mg}$ dose, and the median OS and progression-free survival were in the ranges observed in phase III trials [15]. In the ReDOS study, the dose-escalation group achieved cycle 3 of treatment, but the standard-dose group did not [8]. The results of these studies indicate that optimizing the initial dose is associated with outcome and toxicity, although a relationship between cumulative dose and outcome was not reported. Furthermore, schedule adjustments or discontinuation/restarting, which often occur in real-world settings, were not considered except for the CORRELATE study. Our study shows that cumulative dose until the second cycle in a real-world setting is associated with OS. The association was not statistically significant with the initial dose distribution divided based on median total dose, whereas initial dose was extracted as a prognostic factor in the multivariate analysis. These results indicate that the initial dose should not be decreased arbitrarily and that an individualized starting dose should be considered, consistent with other studies.

The respective incidences of HFSR, liver dysfunction, and hypertension were $80 \%, 31 \%$, and $60 \%$ in the Japanese population in the CORRECT study [4], in contrast to $93.1 \%, 25.5 \%$, and $35.2 \%$, respectively, in this study. The frequency of hypertension in this study was lower than previously reported, whereas that of HFSR was higher. The rates of adverse events of $\geq$ grade 3 were similar to other studies. In groups separated by median total dose, all grades of HFSR were statistically significant, although the frequency of HFSR was generally over $90 \%$ in both groups. These results indicate that HFSR is likely to occur in $\mathrm{mCRC}$ patients treated with regorafenib. The data also indicate that the incidences of skin rash and emergency hospitalization in patients with a total dose until the second cycle $<3180 \mathrm{mg}$ are clearly higher than in patients in the other group. The results show that skin rash and emergency hospitalization are direct causes of discontinuation or dose reduction. It is therefore important to identify patients who are likely to develop severe adverse effects.

Many researchers have examined ways to optimize the dose of regorafenib, but there are no significant real-world data available. We assessed adherence to regorafenib in order to examine real-world doses. It 
has not been previously reported that cumulative dose is associated with survival time in view of realworld adherence data. Our study indicates that total dose until the second cycle $\geq 3180 \mathrm{mg}$ prolongs OS. This value may represent a cut-off point. A regorafenib initial dose of $80 \mathrm{mg}$ continuing until second cycle at the standard schedule would lead to a cumulative dose of $3360 \mathrm{mg}$ in the absence of discontinuation or dose reduction. That is the indicator for regorafenib treatment design in terms of dose escalation, dose reduction, or schedule adjustment.

Since regorafenib was approved, many studies have examined whether pharmacokinetic and pharmacodynamic parameters such as dose setting are associated with efficacy or adverse events. In general, regorafenib is metabolized by cytochrome P450 3A4 in the liver to its active metabolites, M-2 and $M-5$. Kubota et al examined the area under the unbound plasma concentration-time curve (AUCu) for these compounds [16]. Higher AUCu values for M-2 and M-5 on day 1 were associated with significantly shorter progression-free survival than higher AUCu values for total plasma or unchanged drug. Moreover, the relative dose intensity during cycle 1 in patients with higher AUCu values for M-2 or M-5 was lower than that for patients with lower AUCu values. These results suggest that the standard dose was too high and that active metabolites played a significant role in patients' decisions whether to continue treatment. In terms of genetic factors, Kubota et al reported a significant association between the ABCG2 421A/C genotype and AUCu values for the active metabolites, whereas another study reported that other genetic factors were not associated with regorafenib pharmacokinetics [17]. Thus, whether genetic factors actually affect regorafenib efficacy and toxicity remains unclear and should be examined in future studies.

There were three major limitations to this study. The first limitation was the retrospective single-institution design, which caused us to overlook some clinical data or consider selection bias, as our focus was on real-world data regarding adherence to regorafenib. Our results were thus not completely clear. Therefore, prospective analyses should be conducted in the future. The second limitation involved the outcome measures used. It is possible that OS was affected by prior chemotherapy or other patient factors, even though we used a multivariate analysis and minimized confounders as much as possible. The third limitation involved the number of cases. Although the study included patients over a 5-year period, we were not able to calculate the appropriate the number of cases to include, which could have caused us to over- or underestimate our results.

\section{Conclusions}

The cumulative dose of regorafenib until the second cycle in patients with $\mathrm{mCRC}$ is associated with drug efficacy. It is important to determine the optimal regorafenib dose in individual mCRC patients in order to avoid discontinuation or dose reduction, as data regarding regorafenib pharmacokinetics and the effects of genetic factors are inadequate.

\section{Abbreviations}


OS: Overall survival; mCRC: metastatic colorectal cancer; HFSR: hand-foot skin reaction; POC:

Pharmaceutical Outpatient Clinic; ECOG: Eastern Cooperative Oncology Group; PS: Performance status; FTD/TPI: trifluridine/tipiracil.; NCI-CTCAE: National Cancer Institute Common Terminology Criteria for Adverse Events; HR: hazard ratio; AUCu: area under the unbound plasma concentration-time curve

\section{Declarations}

\section{Ethics approval and consent to participate}

The study was approved by the Institutional Review Board of the Cancer Institute Hospital based on biomedical research guidelines specified in the Declaration of Helsinki (approval number 2018-1239). Since the retrospective, the need for informed consent was waived by the Institutional Review Board of the Cancer Institute Hospital. Authors uploaded the information about this study and patients' right to the participating institution's websites.

\section{Consent for publication}

Not applicable. This is just the same as described above

\section{Availability of data and materials}

The data and materials are available from the corresponding author on reasonable request.

\section{Competing interests}

K. Yamaguchi has received speaking honoraria from Taiho Pharmaceutical, Chugai Pharmaceutical, Merk Serono, Takeda Pharmaceutical, Yakult, Bayer, Ono Pharmaceutical, Eli Lilly, Sanofi, and Bristol-Myers Squibb; has received research grants from MSD, Ono Pharmaceutical, Sumitomo Dainippon Pharma, Taiho Pharmaceutical, Daiichi Sankyo, Eli Lilly, Gilead Sciences, and Yakult; and has had a consulting or advisory role for Bristol-Myers Squibb. The other authors declare that they have no conflicts of interest.

\section{Funding}

None.

\section{Authors' contributions}

Conceptualization and study design: $\mathrm{MH}, \mathrm{KK}$; Writing an original draft: $\mathrm{MH}$; Review and editing: $\mathrm{KK}, \mathrm{TW}$, KK, ES, KS; Supervison: KY, TH; Investigation of data: MH, KK, TW, KK, TA, YN; and All authors read and approved the final manuscript.

\section{Acknowledgments}

Not applicable. 
Author details

${ }^{1}$ Department of Pharmacy, Cancer Institute Hospital, Japanese Foundation for Cancer Research, 3-10-6 Ariake, Koto-ku, Tokyo 135-8550, Japan

2 Department of Gastroenterological Chemotherapy, Cancer Institute Hospital, Japanese Foundation for Cancer Research, 3-8-31 Ariake, Koto-ku, Tokyo 135-8550, Japan

${ }^{3}$ Section for Practical Education, Hoshi University School of Pharmacy and Pharmaceutical Sciences, 24-41 Ebara, Shinagawa-ku, Tokyo 142-8501, Japan

\section{References}

1. Ferlay J, Colombet M, Soerjomataram I, Mathers C, Parkin DM, Piñeros M, Znaor A, Bray F: Estimating the global cancer incidence and mortality in 2018: GLOBOCAN sources and methods. International journal of cancer 2019, 144(8):1941-1953.

2. Wilhelm SM, Dumas J, Adnane L, Lynch M, Carter CA, Schütz G, Thierauch KH, Zopf D: Regorafenib (BAY 73-4506): a new oral multikinase inhibitor of angiogenic, stromal and oncogenic receptor tyrosine kinases with potent preclinical antitumor activity. International journal of cancer 2011, 129(1):245-255.

3. Grothey A, Van Cutsem E, Sobrero A, Siena S, Falcone A, Ychou M, Humblet Y, Bouché O, Mineur L, Barone $C$ et al: Regorafenib monotherapy for previously treated metastatic colorectal cancer (CORRECT): an international, multicentre, randomised, placebo-controlled, phase 3 trial. Lancet (London, England) 2013, 381(9863):303-312.

4. Yoshino T, Komatsu Y, Yamada Y, Yamazaki K, Tsuji A, Ura T, Grothey A, Van Cutsem E, Wagner A, Cihon $\mathrm{F}$ et al: Randomized phase III trial of regorafenib in metastatic colorectal cancer: analysis of the CORRECT Japanese and non-Japanese subpopulations. Investigational new drugs 2015, 33(3):740-750.

5. Adenis A, de la Fouchardiere C, Paule B, Burtin P, Tougeron D, Wallet J, Dourthe LM, Etienne PL, Mineur L, Clisant $S$ et al: Survival, safety, and prognostic factors for outcome with Regorafenib in patients with metastatic colorectal cancer refractory to standard therapies: results from a multicenter study (REBECCA) nested within a compassionate use program. BMC cancer 2016, 16:412.

6. Yamaguchi K, Komatsu Y, Satoh T, Uetake H, Yoshino T, Nishida T, Yamazaki N, Takikawa H, Morimoto T, Chosa $\mathrm{M}$ et al: Large-Scale, Prospective Observational Study of Regorafenib in Japanese Patients with Metastatic Colorectal Cancer in a Real-World Clinical Setting. The oncologist 2019, 24(7):e450-e457.

7. Van Cutsem E, Martinelli E, Cascinu S, Sobrero A, Banzi M, Seitz JF, Barone C, Ychou M, Peeters M, Brenner B et al: Regorafenib for Patients with Metastatic Colorectal Cancer Who Progressed After Standard Therapy: Results of the Large, Single-Arm, Open-Label Phase IIlb CONSIGN Study. The oncologist 2019, 24(2):185-192. 
8. Bekaii-Saab TS, Ou FS, Ahn DH, Boland PM, Ciombor KK, Heying EN, Dockter TJ, Jacobs NL, Pasche $\mathrm{BC}$, Cleary JM et al: Regorafenib dose-optimisation in patients with refractory metastatic colorectal cancer (ReDOS): a randomised, multicentre, open-label, phase 2 study. The Lancet Oncology 2019, 20(8):1070-1082.

9. Suzuki T, Sukawa Y, Imamura CK, Masuishi T, Satake H, Kumekawa Y, Funakoshi S, Kotaka M, Horie Y, Kawai S et al: A Phase II Study of Regorafenib With a Lower Starting Dose in Patients With Metastatic Colorectal Cancer: Exposure-Toxicity Analysis of Unbound Regorafenib and Its Active Metabolites (RESET Trial). Clinical colorectal cancer 2020, 19(1):13-21.e13.

10. Institute NC: Common Terminology Criteria for Adverse Events (CTCAE) Version 4.0. May 28, 2009 (v4.03: June 14, 2010).

11. Kawakami K, Wakatsuki T, Soejima A, Kobayashi K, Yokokawa T, Aoyama T, Suzuki K, Suenaga M, Yamaguchi K, Inoue A et al: Factors Associated With Regorafenib Adherence With Metastatic Colorectal Cancer. Patient preference and adherence 2019, 13:1745-1750.

12. Rizzo A, Nannini M, Novelli M, Dalia Ricci A, Scioscio VD, Pantaleo MA: Dose reduction and discontinuation of standard-dose regorafenib associated with adverse drug events in cancer patients: a systematic review and meta-analysis. Therapeutic advances in medical oncology 2020, 12:1758835920936932.

13. Grothey A, George S, van Cutsem E, Blay JY, Sobrero A, Demetri GD: Optimizing treatment outcomes with regorafenib: personalized dosing and other strategies to support patient care. The oncologist 2014, 19(6):669-680.

14. Lam KO, Lee KC, Chiu J, Lee VH, Leung R, Choy TS, Yau T: The real-world use of regorafenib for metastatic colorectal cancer: multicentre analysis of treatment pattern and outcomes in Hong Kong. Postgraduate medical journal 2017, 93(1101):395-400.

15. Ducreux M, Petersen LN, Öhler L, Bergamo F, Metges JP, de Groot JW, Wang JY, García Paredes B, Dochy E, Fiala-Buskies $S$ et al: Safety and effectiveness of regorafenib in patients with metastatic colorectal cancer in routine clinical practice in the prospective, observational CORRELATE study. European journal of cancer (Oxford, England : 1990) 2019, 123:146-154.

16. Kubota Y, Fujita KI, Takahashi T, Sunakawa Y, Ishida H, Hamada K, Ichikawa W, Tsunoda T, Shimada K, Masuo Y et al: Higher Systemic Exposure to Unbound Active Metabolites of Regorafenib Is Associated With Short Progression-Free Survival in Colorectal Cancer Patients. Clinical pharmacology and therapeutics 2020.

17. Maeda A, Irie K, Ando H, Hasegawa A, Taniguchi H, Kadowaki S, Muro K, Tajika M, Aoki M, Inaguma K et al: Associations among regorafenib concentrations, severe adverse reactions, and ABCG2 and OATP1B1 polymorphisms. Cancer chemotherapy and pharmacology 2019, 83(1):107-113.

\section{Tables}

Table 1. Patient characteristics 


\begin{tabular}{|c|c|c|}
\hline Characteristic & No. of patients $(n=176)$ & $(\%)$ \\
\hline \multicolumn{3}{|l|}{ Age } \\
\hline$\geq 65 /<65$ years & $76 / 100$ & $43.2 / 56.8$ \\
\hline \multicolumn{3}{|l|}{ Gender } \\
\hline male/female & $94 / 82$ & $53.4 / 46.6$ \\
\hline \multicolumn{3}{|l|}{ Performance status } \\
\hline 0/1/2/unknown & $89 / 73 / 3 / 11$ & $50.6 / 41.1 / 1.7 / 6.3$ \\
\hline \multicolumn{3}{|l|}{ Primary site } \\
\hline colon & 105 & 59.7 \\
\hline rectum & 58 & 33 \\
\hline cecum & 9 & 5.1 \\
\hline appendix & 4 & 2.2 \\
\hline \multicolumn{3}{|l|}{ Adjuvant chemotherapy } \\
\hline yes/no & $52 / 124$ & $29.5 / 70.5$ \\
\hline \multicolumn{3}{|l|}{ Site of primary tumor } \\
\hline left/right & $122 / 54$ & $69.3 / 30.7$ \\
\hline \multicolumn{3}{|l|}{ KRAS mutations } \\
\hline wild type/mutant/unknown & $83 / 92 / 1$ & $47.1 / 52.3 / 0.6$ \\
\hline \multicolumn{3}{|l|}{ Number of metastatic sites } \\
\hline$\leq 2 / \geq 3$ & $103 / 73$ & $58.5 / 41.5$ \\
\hline \multicolumn{3}{|l|}{ Metastatic site } \\
\hline peritoneal/liver/lung & $55 / 118 / 106$ & $31.3 / 67 / 60.2$ \\
\hline \multicolumn{3}{|l|}{ Number of prior chemotherapies } \\
\hline$\leq 2 / 3 / 4$ & $59 / 86 / 31$ & $33.5 / 48.9 / 17.6$ \\
\hline \multicolumn{3}{|l|}{ Prior chemotherapy } \\
\hline fluoropyrimidine & 176 & 100 \\
\hline irinotecan & 174 & 98.9 \\
\hline oxaliplatin & 175 & 99.4 \\
\hline bevacizumab & 163 & 92.6 \\
\hline
\end{tabular}


Regorafenib initial dose (mg)

$160 / 120 / 80 / 40$

$122 / 43 / 10 / 1$

$69.3 / 24.4 / 5.7 / 0.6$

Anti-EGFR: cetuximab, panitumumab

Table 2. Multivariate analysis of prognostic factors

\begin{tabular}{|c|c|c|c|c|}
\hline Variate & & $\begin{array}{l}\text { Median survival (95\% } \\
\mathrm{Cl})\end{array}$ & $\begin{array}{l}\text { Hazard ratio }(95 \% \\
\mathrm{Cl})\end{array}$ & $P$ value \\
\hline \multirow[t]{2}{*}{$\begin{array}{l}\text { Total dose until second } \\
\text { cycle }\end{array}$} & $\begin{array}{l}\geq 3180 \\
\mathrm{mg}\end{array}$ & $7.61(6.41-8.81)$ & 1 & 0.003 \\
\hline & $<3180 \mathrm{mg}$ & $5.84(4.56-7.12)$ & $1.71(1.20-2.44)$ & \\
\hline \multirow[t]{2}{*}{ Age (years) } & $\geq 65$ & $7.08(5.71-8.46)$ & 1 & $<0.001$ \\
\hline & $<65$ & $6.43(4.96-7.90)$ & $1.96(1.36-2.86)$ & \\
\hline \multirow[t]{3}{*}{ Performance status } & 0 & $8.00(6.94-9.07)$ & 1 & 0.001 \\
\hline & 1 & $5.90(4.73-7.08)$ & $1.81(1.28-2.57)$ & \\
\hline & 2 & $1.57(0.89-2.26)$ & & \\
\hline \multirow[t]{2}{*}{ Hand-foot skin reaction } & Yes & $6.69(5.58-7.80)$ & & 0.325 \\
\hline & No & $5.80(1.67-9.94)$ & & \\
\hline \multirow[t]{2}{*}{ Number of metastatic sites } & $\leq 2$ & $7.61(6.28-8.94)$ & & 0.402 \\
\hline & $\geq 3$ & $6.13(4.40-7.86)$ & & \\
\hline \multirow[t]{2}{*}{ Hepatic metastasis } & Yes & $5.71(4.86-6.55)$ & $2.86(1.90-4.30)$ & $<0.001$ \\
\hline & No & $10.8(6.99-14.5)$ & 1 & \\
\hline \multirow[t]{2}{*}{ Regorafenib initial dose } & $160 \mathrm{mg}$ & $7.34(6.02-8.67)$ & 1 & 0.01 \\
\hline & $\leq 120 \mathrm{mg}$ & $6.10(4.70-7.50)$ & $1.71(1.14-2.58)$ & \\
\hline
\end{tabular}

Table 3. Patient characteristic between groups 
Characteristics

Age (years)

$\geq 65 /<65$

Gender

male/female

Performance status

0/1/2/unknown

Primary site

colon/rectum/cecum/appendix

Adjuvant chemotherapy

yes/no

$20 / 71$

Site of primary tumor

left/right

$62 / 29$

KRAS mutations

wild type/mutant/unknown

Number of metastatic sites

$\leq 2 / \geq 3$

$55 / 36$

$47 / 44 / 0$

$36 / 48 / 1$

$54 / 23 / 3 / 5$

$44 / 35 / 1 / 5$

Total dose until second $P$

cycle $<3180 \mathrm{mg}(\mathrm{n}=85) \quad$ value

0.259

$33 / 52$

$37 / 48$

0.011

$57 / 34$

0.958 *

$48 / 38 / 2 / 3$

$51 / 35 / 1 / 4$

0.023

$32 / 53$

0.724

$60 / 25$

$0.257 *$

Metastatic site

peritoneal

25

62

56

30

0.263

liver

lung

56

55

0.201

50

0.713

Use of antibody drug

bevacizumab

83

80

0.461

anti-EGFR

45

34

0.208

Regorafenib initial dose (mg)

$160 / \leq 120$

$65 / 26$

$57 / 28$

Sequence of chemotherapy

FTD/TPI after regorafenib
0.53

$0.877 *$ 
regorafenib after FTD/TPI

other

26

41

FTD/TPI: trifluridine/tipiracil.

Statistical analysis: characteristics compared by Pearson's chi-square test (or *Fisher's exact test).

Table 4. Adverse events related to regorafenib

Total dose until second cycle $\geq 3180 \mathrm{mg}(\%)(\mathrm{n}=$ 91)
Total dose until second cycle $<3180 \mathrm{mg}(\%)(\mathrm{n}=85) \quad$ value

\begin{tabular}{|c|c|c|c|c|}
\hline & & $\begin{array}{l}\text { Total dose until second } \\
\text { cycle } \geq 3180 \mathrm{mg}(\%)(\mathrm{n}= \\
91)\end{array}$ & $\begin{array}{l}\text { Total dose until second } \\
\text { cycle <3180 mg }(\%)(n=85)\end{array}$ & $\begin{array}{l}P \\
\text { value }\end{array}$ \\
\hline \multirow[t]{2}{*}{$\begin{array}{l}\text { Hand-foot skin } \\
\text { reaction }\end{array}$} & $\begin{array}{l}\text { All } \\
\text { grades }\end{array}$ & $81(89.0)$ & $83(97.6)$ & 0.01 \\
\hline & $\begin{array}{l}\geq \\
3\end{array}$ & $22(24.1)$ & $23(27.0)$ & 0.661 \\
\hline \multirow[t]{2}{*}{$\begin{array}{l}\text { Liver } \\
\text { dysfunction }\end{array}$} & $\begin{array}{l}\text { All } \\
\text { grades }\end{array}$ & 39 (42.9) & $26(30.6)$ & 0.092 \\
\hline & $\begin{array}{l}\geq \text { Grade } \\
3\end{array}$ & $4(4.4)$ & $7(8.2)$ & 0.293 \\
\hline \multirow[t]{2}{*}{ Hypertension } & $\begin{array}{l}\text { All } \\
\text { grades }\end{array}$ & $28(30.7)$ & $34(40.0)$ & 0.2 \\
\hline & $\begin{array}{l}\geq \\
3\end{array}$ & $4(4.4)$ & $14(16.5)$ & 0.008 \\
\hline \multirow[t]{2}{*}{ Skin rash } & $\begin{array}{l}\text { All } \\
\text { grades }\end{array}$ & $7(7.7)$ & $17(20.0)$ & 0.017 \\
\hline & $\begin{array}{l}\geq \text { Grade } \\
3\end{array}$ & $3(3.3)$ & $11(12.9)$ & 0.018 \\
\hline $\begin{array}{l}\text { Emergency } \\
\text { hospitalization }\end{array}$ & & $5(5.5)$ & $16(18.8)$ & 0.006 \\
\hline
\end{tabular}

Statistical analysis: patient characteristics compared by Pearson's chi-square test. 


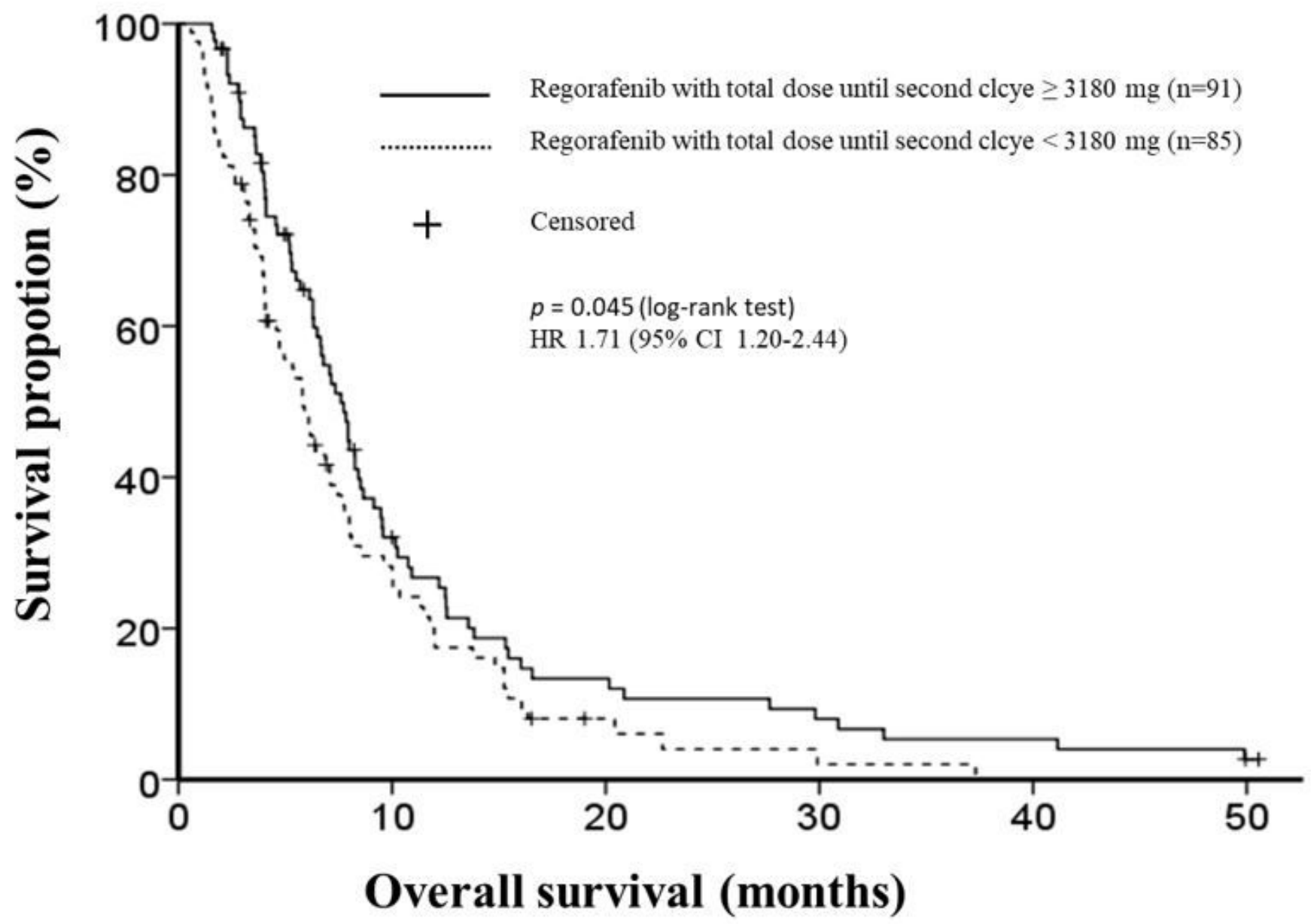

Figure 1

Overall survival between groups based on median total dose. 


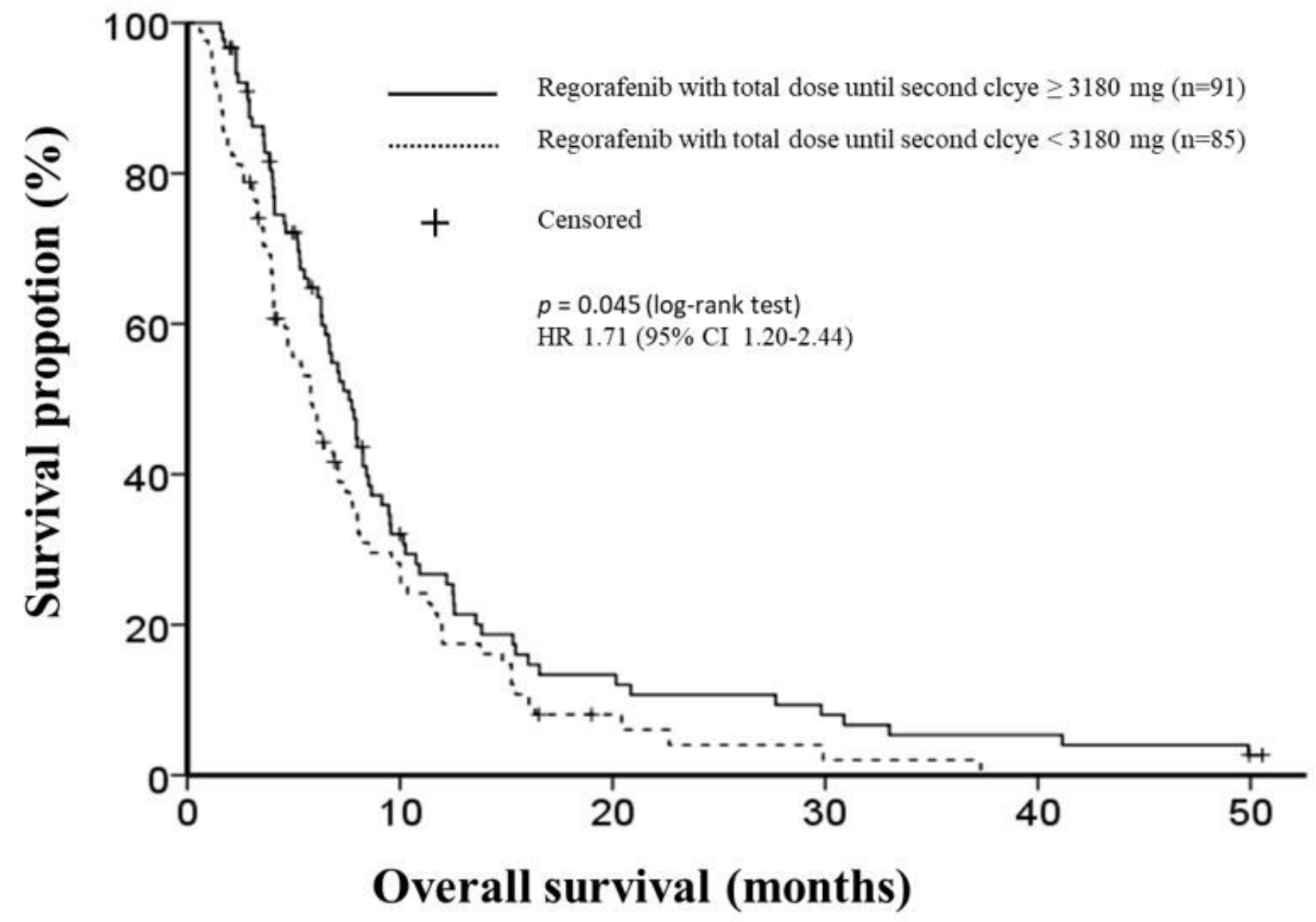

Figure 1

Overall survival between groups based on median total dose.

\section{Supplementary Files}

This is a list of supplementary files associated with this preprint. Click to download.

- supplementarytable.docx

- supplementarytable.docx 